\title{
Diferentes arranjos de semeadura para a cultivar de amendoim BRS 423 OL nas condições de Campo Verde-MT
}

Submetido - 07 jul. 2020

Aprovado - 10 set. 2020

Publicado - 14 out. 2020

\section{http://dx.doi.org/10.17648/sas.v1i2.10}

Felipe Gabriel Giron (D)

Discente de Agronomia do IFMT Campus São Vicente - Centro de Referência de Campo Verde, Campo Verde, MT, e-mail: felipegiron99@outlook.com.

Jair Heuert (D)

Programa de Melhoramento do Amendoim - Embrapa, Santo Antônio de Goiás, GO, e-mail: jair.heuert@embrapa.br.

Alexandre Caetano Perozini (iD

Docente do IFMT Campus São Vicente - Centro de Referência de Campo Verde, Campo Verde, MT, e-mail: alexandre.perozini@svc.ifmt.edu.br.

Maxuel Fellipe Nunes Xavier ${ }^{\text {DD }}$

Discente de Agronomia do IFMT Campus São Vicente - Centro de Referência de Campo Verde, Campo Verde, MT, e-mail: maxuelfellipe90@gmail.com.

\section{Taís de Moraes Falleiro Suassuna ${ }^{D}$}

Programa de Melhoramento do Amendoim - Embrapa, Santo Antônio de Goiás, GO, e-mail: tais.suassuna@embrapa.br.

\section{RESUMO}

O objetivo foi avaliar diferentes arranjos de semeadura de amendoim com a cultivar BRS 423 $O L$, nas condições de Campo Verde-MT. O experimento foi desenvolvido no ano agrícola 2019/20 com semeadura manual no dia 12/11/2019, na área experimental do Instituto Federal de Educação, Ciência e Tecnologia de Mato Grosso - Campus São Vicente, Centro de Referência de Campo Verde, localizado no município de Campo Verde - MT. O delineamento experimental foi em blocos casualizados, com cinco repetições. Os tratamentos foram diferentes arranjos de semeadura: T1 - População de 222.222 plantas.ha-1 no espaçamento de 0,90 m entre linhas simples e 20 plantas. $\mathrm{m}^{-1}$, T2 - População de 222.222 plantas.ha-1 no espaçamento de 0,73 $\mathrm{m} \mathrm{x}$ $0,17 \mathrm{~m}$ entre linhas duplas e 20 plantas. $\mathrm{m}^{-1}$ e T3 - População de 214.285 plantas.ha-1 no $^{-1}$ nostas espaçamento de 0,70 m entre linhas simples e 15 plantas. $\mathrm{m}^{-1}$. As parcelas eram compostas por duas linhas de três metros de comprimento, com intervalo entre parcelas de dois metros e parcela útil 5,4 $\mathrm{m}^{2}$. Foram avaliados massa de 100 grãos e produtividade de vagens, observando diferença significativa nas duas variáveis. As maiores produtividades foram obtidas nos arranjos de população 222.222 plantas.ha-1 no espaçamento 0,73 $\mathrm{m} \times$ 0,17 m entre linhas duplas e 20 plantas. $m^{-1}$ e população 214.285 plantas.ha-1 no espaçamento $0,70 \mathrm{~m}$ entre linhas simples e 15 plantas. $\mathrm{m}^{-1}$, ambos com produtividades superiores a $5.400 \mathrm{~kg} \cdot \mathrm{ha}^{-1}$.

Palavras-chave: Arachis hypogaea L.; Programa de Melhoramento; Espaçamentos; Amendoim em Mato Grosso.

\section{Different sowing arrangements for the BRS 423 OL peanut cultivar in Campo Verde-MT conditions}

\section{ABSTRACT}

The objective was to evaluate different peanut sowing arrangements with the cultivar BRS 423 $O L$, under the conditions of Campo Verde-MT. The experiment was carried out in the agricultural year 2019/20 with manual seeding on 12/11/2019, in the experimental area of the 
Federal Institute of Education, Science and Technology of Mato Grosso - São Vicente Campus, Campo Verde Reference Center, located in the municipality of Campo Verde - MT. The experimental design was in randomized blocks, with five replications. The treatments were different sowing arrangements: T1 - Population of 222,222 plants.ha-1 in the spacing of $0.90 \mathrm{~m}$ between single lines and 20 plants. $\mathrm{m}^{-1}$, T2 - Population of 222,222 plants.ha-1 in the spacing of $0.73 \mathrm{mx} 0.17 \mathrm{~m}$ between double lines and 20 plants. $\mathrm{m}^{-1}$ and T3 - Population of 214,285 plants.ha1 in the spacing of $0.70 \mathrm{~m}$ between single lines and 15 plants. $\mathrm{m}^{-1}$. The plots consisted of two lines three meters long, with an interval between plots of two meters and a useful plot of $5.4 \mathrm{~m}^{2}$. Mass of 100 grains and pod yield were evaluated, observing a significant difference in the two variables. The highest yields were obtained in the population arrangements 222,222 plants.ha ${ }^{-1}$ in the spacing $0.73 \mathrm{mx} 0.17 \mathrm{~m}$ between double lines and 20 plants. $\mathrm{m}^{-1}$ and the population 214,285 plants.ha-1 in the spacing $0.70 \mathrm{~m}$ between simple lines and 15 plants. $\mathrm{m}^{-1}$, both with yields above $5,400 \mathrm{~kg} \cdot \mathrm{ha}^{-1}$.

Keywords: Arachis hypogaea L.; Improvement Program; Spacing; Peanut in Mato Grosso.

\title{
Diferentes arreglos de siembra para el cultivar de maní BRS 423 OL en condiciones de Campo Verde-MT
}

\begin{abstract}
RESUMEN
El objetivo fue evaluar diferentes arreglos de siembra de maní con el cultivar BRS 423 OL, bajo las condiciones de Campo Verde-MT. El experimento se llevó a cabo en el año agrícola $2019 / 20$ con siembra manual el 11/12/2019, en el área experimental del Instituto Federal de Educación, Ciencia y Tecnología de Mato Grosso - Campus São Vicente, Centro de Referencia Campo Verde, ubicado en el municipio de Campo Verde - MT. El diseño experimental fue en bloques al azar, con cinco repeticiones. Los tratamientos fueron diferentes arreglos de siembra: T1 Población de 222.222 plantas.ha-1 en el espacio de 0,90 m entre líneas simples y 20 plantas. $\mathrm{m}^{-1}$, T2 - Población de 222.222 plantas. ha-1 en el espacio de 0,73 m x 0,17 m entre líneas dobles y 20 plantas. $m^{-1}$ y $T 3$ - Población de 214.285 plantas.ha ${ }^{-1}$ en el espacio de 0,70 m entre líneas simples y 15 plantas. $m^{-1}$. Las parcelas consistieron en dos líneas de tres metros de largo, con un intervalo entre parcelas de dos metros y una parcela útil de 5,4 $\mathrm{m}^{2}$. Se evaluaron masas de 100 granos y rendimiento de vaina, observando una diferencia significativa en las dos variables. Los rendimientos más altos se obtuvieron en los arreglos de población 222.222 plantas.ha ${ }^{-1}$ en el espacio de 0,73 m x 0,17 m entre líneas dobles y 20 plantas. $\mathrm{m}^{-1}$ y la población 214.285 plantas.ha-1 en el espacio de 0,70 m entre líneas simples y 15 plantas. $m^{-1}$, ambas con rendimientos superiores a $5.400 \mathrm{~kg} \cdot \mathrm{ha}^{-1}$.
\end{abstract}

Palabras clave: Arachis hypogaea L.; Programa de Mejoramiento; Espaciado; Maní em Mato Grosso.

\section{Introdução}

A densidade populacional é o número de plantas por unidade de área, obtido por meio de arranjo de espaçamento entre linhas e número de plantas por metro. A população de plantas influência diretamente nos componentes de produção de uma cultura, principalmente na produtividade (NAKAGAWA et al., 1983). A densidade pode causar variação na massa de 100 grãos, podendo ser reduzida conforme o aumento de número de plantas por hectare (GOPALASWAMY et al., 1979). 
A utilização de espaçamento adequado contribui em diversos fatores na planta, como: incremento produtivo, controle de plantas infestantes, otimização na absorção e uso da luz, água, nutrientes. Além de influenciar em caráter econômico, sobre o maior ou menor consumo de sementes na lavoura (NAKAGAWA et al., 1994).

Os espaçamentos equidistantes, ou seja, em linhas simples que possuam a mesma distância entre si, podem resultar em incrementos na: produtividade, cobertura do solo, índice de área foliar, interceptação luminosa pelo dossel da planta e taxa de crescimento (JAAFFAR; GARDNER, 1988).

$\mathrm{Na}$ Argentina utilizam-se o espaçamento em linhas simples de 0,70 m (GAMBA; PEDELINI, 2009). Portanto, nos Estados Unidos é mais usado linhas duplas de 0,73 $\mathrm{m} \times 0,17 \mathrm{~m}$, sendo também um espaçamento usado no cultivo de amendoim no Brasil (HEUERT et al., 2017). O presente trabalho teve como objetivo avaliar diferentes arranjos de semeadura de amendoim com a cultivar BRS 423 OL, nas condições de Campo Verde-MT.

\section{Material e métodos}

O experimento foi desenvolvido no ano agrícola 2019/20 com semeadura manual no dia 12/11/2019, na área experimental do Instituto Federal de Educação, Ciência e Tecnologia de Mato Grosso - Campus São Vicente, Centro de Referência de Campo Verde, localizado no município de Campo Verde - MT.

O delineamento experimental utilizado foi em blocos casualizados, com cinco repetições. Os tratamentos foram diferentes arranjos de semeadura: T1 - População de 222.222 plantas.ha ${ }^{-1}$ no espaçamento de 0,90 m entre linhas simples e 20 plantas. $\mathrm{m}^{-1}$, T2 - População de 222.222 plantas.ha-1 no espaçamento de $0,73 \mathrm{~m} \times 0,17 \mathrm{~m}$ entre linhas duplas e 20 plantas. $\mathrm{m}^{-1}$ e T3 - População de 214.285 plantas. ha- ${ }^{-1}$ no espaçamento de 0,70 m entre linhas simples e 15 plantas. $\mathrm{m}^{-1}$. A cultivar semeada foi a BRS $423 \mathrm{OL}$, desenvolvida pelo Programa de Melhoramento do Amendoim da 
Embrapa. As parcelas eram compostas por duas linhas de três metros de comprimento, com intervalo entre parcelas de dois metros e parcela útil 5,4 $\mathrm{m}^{2}$.

O manejo fitossanitário da área experimental seguiu as recomendações para a cultura. O manejo fitossanitário foi realizado com controle de plantas daninhas realizado com seis aplicações de herbicida: glifosato (2,0 kg p.c.ha-1) aplicado no dia 11/10/2019, quizalofope-p-etílico $\left(1,0\right.$ L p.c.ha $\left.^{-1}\right)+$ etoxissulfurom $\left(0,05 \mathrm{~kg} \mathrm{p.c.ha}^{-1}\right)$ no dia 22/10/2019 e imazapique $\left(0,14 \mathrm{~kg} \mathrm{p.c.ha}^{-1}\right)$ nos dias 14/12/2019, 23/01/2020, 10/02/2020, 22/02/2020. O manejo de pragas e doenças foi realizado com oito aplicações de inseticida clorfenapir $\left(0,5 \mathrm{~L} \mathrm{p.c.ha}^{-1}\right)$ e fungicidas clorotalonil $(1,5 \mathrm{~L} \mathrm{p.c.ha}$ $\left.{ }^{1}\right)$, pyraclostrobina + epoxiconazol $\left(0,6\right.$ L p.c.ha $\left.{ }^{-1}\right)$ nos dias 14/12/2019, 23/12/2019, 03/01/2020, 13/01/2020, 27/01/2020, 10/02/2020, 22/02/2020 e 05/03/2020.

Com base na necessidade do solo, foi realizado adubação de semeadura de $400 \mathrm{~kg} \cdot \mathrm{ha}^{-1}$ de superfosfato simples (SFS) no sulco de plantio. No tratamento de sementes foi utilizado carbendazim, na dose de $0,1 \mathrm{~L}$ por $100 \mathrm{~kg}$ de sementes. Foram feitas duas aplicações de adubação de cobertura, nos dias 19/12/2019 e 03/01/2020, ambas na dose de $50 \mathrm{~kg} \mathrm{ha}^{-1}$ de cloreto de potássio $(\mathrm{KCl})$ e uma aplicação de gesso agrícola no dia 23/12/2019 na dose de $500 \mathrm{~kg} \cdot \mathrm{ha}^{-1}$.

Realizou-se a colheita aos 120 dias após a emergência (DAE), realizando avaliações de massa de 100 grãos (g) e produtividade de vagens (kg.ha-1 e sacas.alqueire $^{-1}$ ), por meio da pesagem de vagens e grãos, da área de 3,6 $\mathrm{m}^{2}$ (T1 e T2) e 2,8 $\mathrm{m}^{2}$ (T3) centrais da parcela. Os dados obtidos foram submetidos à análise de variância (Teste $F$ ) e as médias dos tratamentos foram comparados pelo teste de Scott-Knott a $5 \%$ de probabilidade, por meio do programa computacional SISVAR 5.6 (FERREIRA, 2019). 


\section{Resultados e discussão}

Houve diferença significativa para a massa de 100 grãos e produtividade de vagens em função dos diferentes arranjos de semeadura de amendoim com a cultivar BRS $423 \mathrm{OL}$ (Tabela 1). As maiores massas de 100 grãos foram obtidas no arranjo com população de 222.222 plantas. ha-1 $^{-1}$ no espaçamento $0,90 \mathrm{~m}$ entre linhas simples e 20 plantas. $\mathrm{m}^{-1}$ (72,5 g). Rizzi et al. (2019), obtiveram massa de 100 grãos de $71,8 \mathrm{~g}$ com o cultivar BRS $423 \mathrm{OL}$ semeado com espaçamento de 0,90 m entre linhas simples, no munícipio de Sorriso-MT.

Portanto, quando utilizada a população de 222.222 plantas. ha $^{-1}$ no

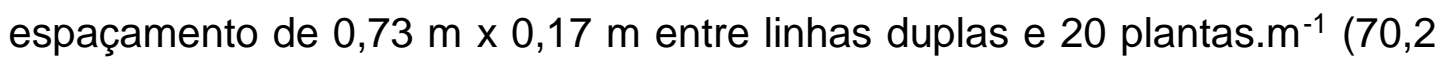
g) e 214.285 plantas.ha ${ }^{-1}$ no espaçamento $0,70 \mathrm{~m}$ entre linhas simples e 15 plantas. $\mathrm{m}^{-1}(69,2 \mathrm{~g})$, obtém-se as menores massas, que não diferem entre si. Observa-se que os diferentes arranjos de semeadura interferem na granulometria do BRS $423 \mathrm{OL}$, nas condições experimentais.

Tabela 1. Massa de 100 grãos (g) e produtividade de vagens $\left(\mathrm{kg}^{-h a^{-1}} \mathrm{e}\right.$ sacas.alqueire ${ }^{-1}$ ) em função de diferentes arranjos de semeadura de amendoim BRS 423 OL. Campo Verde-MT, 2019/20.

\begin{tabular}{|c|c|c|c|c|c|c|}
\hline \multirow{2}{*}{ Espaçamento } & \multicolumn{2}{|c|}{$\begin{array}{l}\text { Número de } \\
\text { plantas }\end{array}$} & \multirow{2}{*}{$\begin{array}{c}\text { Massa } \\
\text { de } \\
100 \\
\text { grãos } \\
(\mathrm{g}) \\
\end{array}$} & \multicolumn{3}{|c|}{ Produtividade de vagens } \\
\hline & metro & hectare & & $\left(\mathrm{kg} \cdot \mathrm{ha}^{-1}\right)$ & $\left(\right.$ sacas.alqueire $^{-1}$ ) & $\begin{array}{c}\text { Incremento } \\
(\%)\end{array}$ \\
\hline $0,90 \mathrm{~m}$ & 20 & 222.222 & $72,5 \mathrm{a}$ & $4.555,2 \mathrm{~b}$ & 440,9 & - \\
\hline $0,73 \mathrm{~m} \times 0,17 \mathrm{~m}$ & $10 \times 10$ & 222.222 & $70,2 \mathrm{~b}$ & $5.539,4 \mathrm{a}$ & 536,2 & 21,6 \\
\hline $0,70 \mathrm{~m}$ & 15 & 214.285 & $69,2 \mathrm{~b}$ & $5.481,2 \mathrm{a}$ & 530,6 & 20,3 \\
\hline C.V. (\%) & - & - & 1,8 & 5,4 & - & - \\
\hline F calculado & - & - & $0,0096^{*}$ & $0,0009^{*}$ & - & - \\
\hline
\end{tabular}

As maiores produtividades de vagens foram obtidas com os arranjos de semeadura com população 222.222 plantas.ha $^{-1}$ no espaçamento $0,73 \mathrm{mx}$

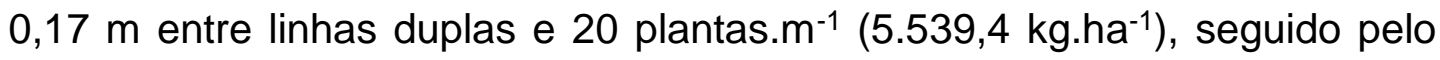
214.285 plantas. ha $^{-1}$ no espaçamento $0,70 \mathrm{~m}$ entre linhas simples e 15 plantas. $\mathrm{m}^{-1} \quad\left(5.481,2 \mathrm{~kg} \cdot \mathrm{ha}^{-1}\right)$, ambos apresentaram produtividades 
superiores a $5.400 \mathrm{~kg} \cdot \mathrm{ha}^{-1}$. O arranjo de população 222.222 plantas.ha-1 no espaçamento $0,90 \mathrm{~m}$ entre linhas simples e 20 plantas. $\mathrm{m}^{-1}$ (4.555,2 kg.ha-1), obteve a menor produtividade e a maior massa de 100 grãos. Observa-se que os arranjos mais produtivos (222.222 plantas. ha-1 no espaçamento 0,73 $\mathrm{m} \times 0,17 \mathrm{~m}$ entre linhas duplas e 20 plantas. $\mathrm{m}^{-1}$ e 214.285 plantas.ha- ${ }^{-1}$ no espaçamento $0,70 \mathrm{~m}$ entre linhas simples e 15 plantas. $\mathrm{m}^{-1}$ ), possuem incrementos de 21,6 e 20,3\%, quando comparados ao arranjo menos produtivo (222.222 plantas.ha-1 no espaçamento 0,90 m entre linhas simples e 20 plantas. $\left.\mathrm{m}^{-1}\right)$.

No entanto, todos os arranjos apresentaram produtividades superiores a estimativa média nacional de $3.553 \mathrm{~kg} \cdot \mathrm{ha}^{-1}$ da Companhia Nacional de Abastecimento (2020), para o cultivo de amendoim primeira safra.

Os arranjos com população 222.222 plantas.ha-1 no espaçamento 0,73 $\mathrm{m} \times 0,17 \mathrm{~m}$ entre linhas duplas e 20 plantas. $\mathrm{m}^{-1}$ e 214.285 plantas.ha- ${ }^{-1}$ no espaçamento $0,70 \mathrm{~m}$ entre linhas simples e 15 plantas. $\mathrm{m}^{-1}$, demonstraram potencial para recomendações de arranjo de semeadura nas condições de Campo Verde-MT. Corroborando com Giron et al. (2019), que descreveram potencial do arranjo 214.285 plantas. $^{-1}{ }^{-1}$ no espaçamento $0,70 \mathrm{~m}$ entre linhas simples e 15 plantas. $\mathrm{m}^{-1}$, com a mesma cultivar para as mesmas condições na safra 2018/19.

Heuert et al. (2018), testando arranjos de semeadura, observaram a população 221.500 plantas.ha-1 no espaçamento $0,70 \mathrm{~m}$ entre linhas simples e 15,5 plantas. $\mathrm{m}^{-1}$ e 222.222 plantas.ha- ${ }^{-1}$ no espaçamento $0,73 \mathrm{~m} \times 0,17 \mathrm{~m}$ entre linhas duplas e 20 plantas. $\mathrm{m}^{-1}$, quando comparadas a 222.222 plantas.ha-1 no espaçamento $0,90 \mathrm{~m}$ entre linhas simples e 20 plantas. $\mathrm{m}^{-1}$, incrementos de 30,0 e $11,2 \%$, respectivamente, utilizando a cultivar BRS 423 OL. Heuert et al. (2019), com o mesmo estudo observou os respectivos incrementos de 24,8 e $10,3 \%$, em relação a população de 222.222 plantas. ha-1 no espaçamento $0,90 \mathrm{~m}$ e 20 plantas. $\mathrm{m}^{-1}$. 


\section{Conclusões}

As maiores produtividades foram obtidas nos arranjos de população 222.222 plantas. ha- ${ }^{-1}$ no espaçamento $0,73 \mathrm{~m} \times 0,17 \mathrm{~m}$ entre linhas duplas $\mathrm{e}$ 20 plantas. $\mathrm{m}^{-1}$ e população 214.285 plantas.ha ${ }^{-1}$ no espaçamento $0,70 \mathrm{~m}$ entre linhas simples e 15 plantas. $\mathrm{m}^{-1}$, ambos com produtividades superiores a $5.400 \mathrm{~kg} \cdot \mathrm{ha}^{-1}$.

\section{Agradecimentos}

Os autores agradecem ao IFMT Campus São Vicente - Centro de Referência de Campo Verde e ao Programa de Melhoramento do Amendoim da Embrapa, vinculado ao projeto (SEG 20.18.01.021.00), localizada em Santo Antônio do Goiás-GO, pelo apoio. 


\section{Referências}

Companhia Nacional de Abastecimento. Acompanhamento da Safra Brasileira de grãos. Safra 2019/20 - Oitavo levantamento, v. 7, n. 8, p. 1 66, maio. 2020. Disponível em: <https://www.conab.gov.br/info-agro/safras>. Acesso em: 6 jun. 2020.

FERREIRA, D. F. SISVAR: um sistema de análise de computador para efeitos fixos projetos de tipo de partida dividida. Revista Brasileira de Biometria, v. 37, n. 4, p. 529-535, 2019.

GAMBA, J. M; PEDELINI, R. Evaluacion del rendimento y calidad de três cultivares de mani. Granoleico, ASEM 484 INTA y ASEM 485 INTA. Xxiv Jornada Nacional de Maní. General Gabrera, Cordoba - Argentina, 2009.

GIRON, F. G.; PEROZINI, A. C.; ARAÚJO, C.; HEUERT, J.; XAVIER, M. F. N.; SUASSUNA, T. M. F. SANTIN, V. Diferentes espaçamentos e densidades populacionais para cultivar de amendoim BRS 423 em Mato Grosso. In: Anais do Encontro Sobre a Cultura do Amendoim, 16., 2019, Jaboticabal. Anais eletrônicos... Campinas: GALOÁ, 2019. Disponível em: $<$ https://proceedings.science/encontro-amendoim-2019/papers/diferentesespacamentos-e-densidades-populacionais-para-cultivar-de-amendoim-brs423-em-mato-grosso. . Acesso em: 6 jun. 2020.

GOPALASWAMY, N.; ELANGOVAN, R.; RAJAH, C. Agronomic and economic optimum plant densities for rainfed groundnut. Indian Journal of Agricultural Sciences, v. 49, n. 1, p. 17-21, 1979.

HEUERT, J.; SOUZA, A. C. A.; OLIVEIRA, B. N.; ZERBATO, C.; COSTA, L. C.; MARTINS, K. B. B.; SUASSUNA, T. M. F.; RIBEIRO, T. C. N.; RIBEIRO, R. P.; SOFIATTI, V. Arranjo de semeadura para cultivares decumbentes precoces de amendoim. In: Anais do Encontro Sobre a Cultura do Amendoim, 14., 2017, Jaboticabal. Anais eletrônicos... Campinas: GALOÁ, 2017. Disponível em: <https://www.embrapa.br/busca-de-publicacoes//publicacao/1085138/arranjo-de-semeadura-para-cultivares-decumbentesprecoces-de-amendoim>. Acesso em: 8 jun. 2020.

HEUERT, J.; SUASSUNA, T. M. F.; MARTINS, K. B. B.; GONÇALVES, W. C. Arranjos com diferentes espaçamentos para cultivares decumbentes precoces de amendoim. In: Anais do Encontro Sobre a Cultura do Amendoim, 15., 2018, Jaboticabal. Anais eletrônicos... Campinas: GALOÁ, 2018. Disponível em: <https://proceedings.science/encontro-amendoim2018/papers/arranjos-com-diferentes-espacamentos-para-cultivaresdecumbentes-precoces-de-amendoim>. Acesso em: 6 jun. 2020.

HEUERT, J.; MARTINS, K. B. B.; XAVIER, M. F. N.; BETIOL, R. A. B.; SUASSUNA, T. M. F. Diferentes espaçamentos para a cultivar de amendoim BRS 423 em Goiás. In: Anais do Encontro Sobre a Cultura do Amendoim, 16., 2019, Jaboticabal. Anais eletrônicos... Campinas: GALOÁ, 2019. Disponível em: <https://proceedings.science/encontro-amendoim- 
2019/papers/diferentes-espacamentos-para-a-cultivar-de-amendoim-brs423-em-goias>. Acesso em: 6 jun. 2020.

JAAFFAR, Z.; GARDNER, F. P. Canopy development, yield and market quality in peanut as affect by genotype and planting pattern. Crop Science, v. 28, n. 2, p. 299-305, 1988.

NAKAGAWA, J.; NOJIMOTO, T.; ROSOLEM, C. A.; ALMEIDA, A. M.; LASCA, D. H. C. Efeitos da densidade de semeadura na produção de vagens de amendoim. Científica, v. 11, n. 1, p. 79-86, 1983.

NAKAGAWA, J.; LASCA, D. C.; NEVES, J. P. S.; NEVES, G. S.; SANCHEZ, S. V.; BARBOSA, V.; SILVA, M. N.; ROSSETO, C. A. V. Efeito na densidade de semeadura na produção de amendoim. Pesquisa Agropecuária Brasileira, v. 29, n. 10, p.1547-1555, 1994.

RIZZI, T. S.; OLIBONE, D.; LODEA, L.; HEUERT, J.; XAVIER, M. F. N.; SUASSUNA, T. M F. Desempenho de cultivares de amendoim na região Médio-Norte Mato-grossense. In: Anais do Encontro Sobre a Cultura do Amendoim, 16., 2019, Jaboticabal. Anais eletrônicos... Campinas: GALOÁ, 2019. Disponível em: <https://proceedings.science/encontro-amendoim2019/papers/desempenho-de-cultivares-de-amendoim-na-regiao-medionorte-mato-grossense>. Acesso em: 6 jun 2020. 